\title{
Developing an Agent-Based Model on How Different Individuals Solve Complex Problems
}

\author{
Ipek Bozkurt \\ University of Houston Clear Lake (United States) \\ Bozkur@ ubcl.edu
}

Received: June 2014

Accepted: December 2014

\section{Abstract:}

Purpose: Research that focuses on the emotional, mental, behavioral and cognitive capabilities of individuals has been abundant within disciplines such as psychology, sociology, and anthropology, among others. However, when facing complex problems, a new perspective to understand individuals is necessary. The main purpose of this paper is to develop an agentbased model and simulation to gain understanding on the decision-making and problem-solving abilities of individuals.

Design/methodology/approach: The micro-level analysis modeling and simulation paradigm Agent-Based Modeling Through the use of Agent-Based Modeling, insight is gained on how different individuals with different profiles deal with complex problems. Using previous literature from different bodies of knowledge, established theories and certain assumptions as input parameters, a model is built and executed through a computer simulation.

Findings: The results indicate that individuals with certain profiles have better capabilities to deal with complex problems. Moderate profiles could solve the entire complex problem, whereas profiles within extreme conditions could not. This indicates that having a strong predisposition is not the ideal way when approaching complex problems, and there should always be a component from the other perspective. The probability that an individual may use these capabilities provided by the opposite predisposition provides to be a useful option. 
Originality/value: The originality of the present research stems from how individuals are profiled, and the model and simulation that is built to understand how they solve complex problems. The development of the agent-based model adds value to the existing body of knowledge within both social sciences, and modeling and simulation.

Keywords: modeling, simulation, agent-based, problem-solving, personality, complex problems

\section{Introduction}

Agent-Based Modeling ( $A B M$ ) has been extensively used outside of the common application environment (engineering, computer science and operations research, among others). These other areas include leadership (Gigliotta, Miglino \& Parisi, 2007), decision-making (Wilson, 2007; Sun \& Naveh, 2004), teamwork (Overwalle \& Heylighen, 2006), sociology (Sallach \& Macal, 2001; Gilbert \& Abbot, 2005; Todd, Billari \& Simao, 2005) and cognition and emotion (Bandura, 2001; Gratch \& Marsella, 2005).

In relation to personality, cognition and psychology, there have been various studies that used modeling and simulation to gain insight and understanding on specific issues that are covered by these bodies of knowledge. Egges, Kshirsagar and Mognenat-Thalmann (2003) conducted a study where personality and emotion of individuals was simulated. Kikuchi and Nakamori (2007) used the Big Five Theory of Personality Psychology factors as rules for a genetic algorithm. In the study conducted by Ghasem-Aghaee and Oren (2007), the agents that are being used have certain personality traits, based on Big Five Personality Theory. Martinez-Miranda and Aldea (2005) have stated that the use of Multi-Agent Systems is beneficial, not only because of the autonomous properties the agents have, but because additional characteristics and agent can take, such as coordination and communication. Hendrickson and McKelvey (in Silverman, Bharathy \& Nye, 2007) state that there is a need for the theories of social science to be computationally formulized as agent models, in order to show that they are analytically adequate.

The Philosophical Profile of the Individual (PPI), i.e. the personality profile that is being modeled and simulated here, has been developed in Bozkurt, Padilla and Sousa-Poza (2007). The detailed premises that are used as the foundation of the profile can be found in that study. Only a brief overview will be presented here to make the readers familiar with the concepts that will be discussed throughout this paper. The focus of this current paper is on developing an agent-based model and simulating it using NetLogo (developed by Uri Wilensky in 1999), and analyzing the results. 


\section{The Philosophical Profile of an Individual}

The Philosophical Profile of the Individual is a model which states that three underlying philosophies, namely Epistemology, Ontology and Teleology, are present within an individual as embedded values that guide how the individual approaches reality, chooses to acquire knowledge, and deals with the future. The constructs of the PPI are useful when individuals tend to solve complex problems. The PPI is built and represented using a three dimensional graphic, shown below in Figure 1 and Figure 2. A three-dimensional representation is used to better address the philosophical profiles. Eight profiles are obtained out of the combination of the paradigms by going through the following steps:

1. Establish the main categories for the axis

- x-axis: Ontology

- y-axis: Epistemology

- z-axis: Teleology

2. Establish orthogonal components for each category

- Ontology: Substantive-Process

- Epistemology: Empiricism-Rationalism

- Teleology: Goal Oriented-No Goal Oriented

3. Construct all possible combinations using all three categories

From the above set-up, a total of eight profiles are obtained. Depending on the dimension, the characteristics and capabilities of the PPI will change.

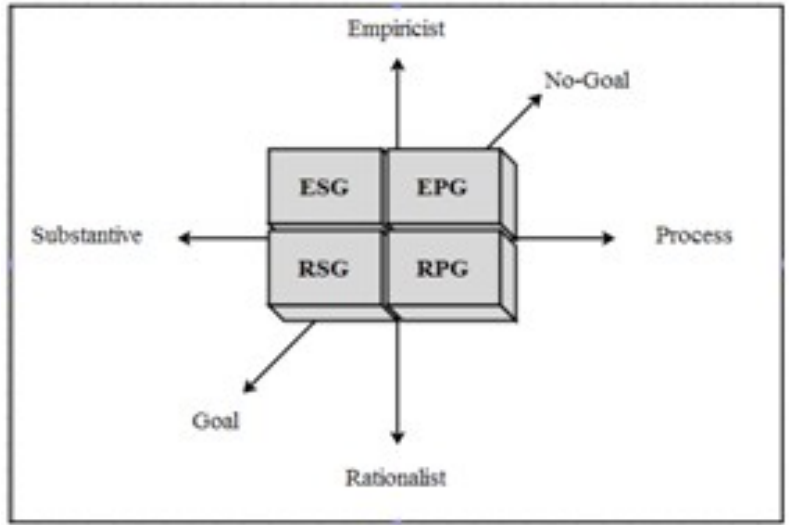

Figure 1. The PPI structure 1

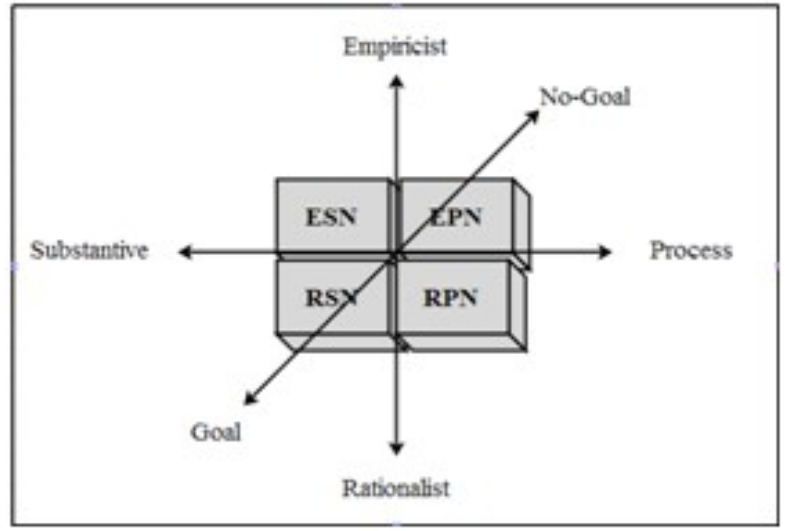

Figure 2. The PPI Structure 2 
Each of the three dimensions is present within an individual, since the dimensions complete each other in an inseparable way. An individual, at any point in time, will have a representation and definition of reality, or the things around himself or herself; the individual will have some means of acquiring information and knowledge about the world surrounding himself or herself; and the individual will act or behave in a certain way for different purposes.

Empiricist-Substantive-Goal (ESG) profile represents PPI1: The individual who fits this profile needs to observe facts, make use of sense data and hard, tangible constructs in order to acquire knowledge about a certain problem. Engineers are a good example for this type of profile. The highly observant nature of engineering is very fitting here, along with the reductionist, predictable and goal-oriented approaches.

Empiricist-Process-Goal (EPG) profile represents PPI2: In the second profile, the epistemological tendency of the individual is again empiricism, where knowledge is acquired through observation, data and facts. The ontological predisposition is from a process philosophy perspective, where the reality is seen as a continuum, meaning that the individual will have a tendency to consider things as a whole. The future is seen as predictable and the individual is goal-oriented.

Rationalist-Substantive-Goal (RSG) profile represents PPI3: An individual with this type of profile seeks knowledge using reasoning and deduction, which is representative of a rationalist epistemological predisposition. Mathematicians would be a good example for this, since a purely theoretical approach to reality and knowledge is dominant in this profile, especially in terms of epistemological arguments.

Rationalist-Process-Goal (RPG) profile represents PPI4: The fourth profile describes an individual who has a tendency towards seeking and gaining knowledge from a rationalist perspective. Managers, for instance, may be a good example for this type of profile. The managers often need to work with deadlines; therefore keeping up with certain goals would be an appropriate choice for them. Also, they often may need to make decisions when empirical data is lacking; therefore a rationalist predisposition would be seen in managers and leaders, since they would follow certain reasoning processes to reach decisions.

Empiricist-Substantive-NoGoal (ESN) profile represents PPI5: This fifth profile presents an individual who has an empiricist tendency from an epistemological stance, meaning that reliance upon observable facts plays an important role for this individual. One needs to have a certain holistic approach in order to better deal with unknown states.

Empiricist-Process-NoGoal (EPN) profile represents PPI6: In the sixth profile, the individual is an empiricist who tends to rely on sense data, observations, measurements, etc. From an ontological perspective, the individual sees reality as a process, as a whole. Some levels of 
military may be an appropriate example for this. When the future is unpredictable, one needs to look at the big picture, but also rely on observations.

Rationalist-Substantive-NoGoal (RSN) profile represents PPI7: This profile seeks knowledge through a deductive approach, using processes such as reasoning and logic to acquire and also create knowledge. An individual with this particular profile feels comfortable dealing with high levels of uncertainty while using discrete concepts that can be generated via imagination, creativity and not necessarily facts.

Rationalist-Process-NoGoal (RPN) profile represents PPI8: The last profile describes an individual who is rationalistic from an epistemological perspective. Leaders and politicians may be good examples for this. When dealing with uncertain situations, one needs to be able to see the big future and make rationalist decisions.

Problem complexity proved to be an intersection of many different bodies knowledge, including problem-solving, complexity and systems theories. Many different characteristics of complex problems were defined by various researchers. Rittel and Weber (1973) defined wicked problems as having no definition, no unique or optimal solution, no time-constraints for testing a solution, no trial and error learning and no truth, meaning that there would be just improvement. Lloyd (1978) described complex problems as having many variables, complex relationships between entities, impracticality in fragmenting the problems, the dynamic nature, and difficulty in establishing cause-and-effect relationship. Steinberg (1983) presented two main characteristics as the number of components, and the problem solver's familiarity with the problem, which also is related to the availability of information on the problem. Funke (1991), who was one of the leading scholars of the German school of complex problems, attributed complexity in problems to the lack of transparency (the ambiguous nature), presence of multiple goals, the connectivity of variables, the dynamic developments within the environment and the problem, and the effects of time constraints. The time issues were also discussed by Augier, Sharig and Vendele (2001) and Quesada, Kintsch and Gomez (2005). Bar-Yam (1993), providing a more systemic perspective, described complexity as the number of components, the relationship among the components, and the information needed on these components. Another perspective in terms of systems complexity came from Flood and Carson (1993), where they stated that the nature of complexity would arise from large number of parts, significant interactions, nonlinearity, and nonholonomic constraints.

From these studies, the most common variables that define complex problems were chosen, which are information availability, ambiguity, time-sensitivity, stability and number of entities. From the above arguments, the following premises are obtained: 


\section{Complex Problem Related Premises:}

- A complex problem is defined by the following properties

- Information available

- Ambiguity

- Time-Sensitivity

- Stability

- Number of entities

- According to the different properties, the problem ranges from simple to complex (Davis, 1969).

- Individuals with different profiles deal with problems differently (Mumford, 1998).

- Information available in a problem (Duncan, 1972; Bar-Yam, 1993; Hood, Logsdon \& Thompson, 1993): This represents any information about the problem that is currently and potentially available to the problem solver. This may be available resources, available hard data, historical data, etc. In complex problems, some information (but not enough) is provided to solve the problem; therefore available information is low, whereas in simple problems, the available information is high.

- Number of entities in a problem (Lloyd, 1978; Steinberg, 1983; Bar-Yam, 1993; Flood \& Carson, 1993): Number of entities is the number of components that come together to formulate the problem. Usually, this means that the more components a problem has the more complex it gets. Therefore, in complex problems, the number of entities is high, whereas in simple problems the number of entities is low.

- Ambiguity in a problem (Lawrence \& Lorsch, 1967; Rittel \& Weber, 1973; Lloyd, 1978; Funke, 1991): An ambiguous problem involves uncertainties. Instead of having a clearly defined problem, there are gray and black areas within the boundaries of a problem. In some cases, the ambiguity may even surface due to the vagueness of the boundaries. The less ambiguous the problem is; a more clear definition exists. This may initially seem related to available information. For instance, can a problem still be ambiguous even though there is a lot of information available about the problem? Yes. The individual may have/or acquire information about a problem, but may not know how to use it, how or where to apply it. Also, when a problem is dynamic, the information an individual has may not be useful after a while, after the problem has changed states.

- Stability in a problem (Swinth, 1971; Lloyd, 1978; Funke, 1991; Quesada et al, 2005): Stability deals with the initial and final states of the problem. A problem may be 
considered to be stable if the conditions of a problem do not change. Complex problems have a tendency to evolve from one state to another. At any time $=t$, the characteristics of the problem may have changed. The desired end-state gets continuously redefined, which means that the problem is dynamic. A high level of stability indicates that the problem is simple.

- Time-Sensitivity in a problem (Lawrence \& Lorsch, 1967; Funke, 1991; Augier et al, 2001; Quesada et al, 2005): The temporal component of the problem is focused on whether the solution of the problem is attached to any deadlines, time restrictions or limitations. For certain types of problems, time may be the most important component. For other problems, even though finding a solution is the ultimate goal, the way in which solutions are found, other emerging aspects of the problem while this process is in effect, may also be significant improvements. This argument can also be supported by whether the problem has a long-time horizon (which in this case the key concern would be effectiveness) or a short-time horizon (which would make the key concern efficiency). This variable also becomes important when all the other factors are put in, for instance, when trying to collect all possible data about a problem may help with accurate problem definition, if the problem has time constraints, this may not be desirable.

\section{Analyzing Theory through Simulation}

Agent-based modeling and simulation has its own theoretical foundations, worldviews and philosophies built through its connection to other fields such as complexity science, systems science, systems dynamics, traditional modeling and simulation, and the social sciences (Macal \& North, 2005). Epstein (1999: page 56) describes ABM as a "powerful tool in the analysis of spatially distributed systems of heterogeneous autonomous actors with bounded information and computing capacity [italics original]". Most social and psychological phenomena occur not as the result of isolated decisions by individuals but rather as the result of repeated interactions between multiple individuals over time (Smith \& Conrey, 2007). Therefore, it is important to choose a specific method that will enable the researcher to observe these repetitive interactions and the patterns that emerge as a result of these interactions.

Smith and Conrey (2007) propose that ABM is an alternative approach to theory building in order to understand dynamic and interactive processes. "One hallmark of ABM is that it typically assumes that the overall system's complexity emerges from the interaction of many very simple components, rather than from great complexity in the behavior of individual agents" (Kauffman, 1995 in Smith and Conrey). Agent-based models are dynamic, expressive and afford immediate feedback (Abrahamson \& Wilensky, 2005). ABM of human behavior is a growing research practice that has shed light on complex dynamic phenomena (e.g., 
Kauffman, 1995; Holland, 1995). Agent-based models are particularly useful for understanding complex phenomena (Abrahamson \& Wilensky, 2005). Agent-Based model will be constructed in the following part.

Epstein (1999) highlights some of the characteristics that make ABM a unique paradigm by addressing certain conditions that are pervasive in managerial conditions. Those conditions are heterogeneity, where all agent populations, similar to individuals, are heterogeneous and may differ in various ways which can change or adapt over time; autonomy, where there is a lack of top-down structure, and therefore agents are autonomous in behavior; explicit space, which is the medium where all the events between agents take place; local interactions, where agents interact with each other, as well as the environment (or the space) itself; and finally bounded rationality, which is a concept that was initially developed by Simon (1972), which states that individuals cannot possess complete knowledge of reality. All of these concepts reflect the flexibility and openness of agent-based modeling when dealing with intangible, real-life constructs such as personality.

Peshkin (1993: page 27) states that "clarifying and understanding complexity [italics from original quote], another outcome of interpretation, is important because most of what we study is truly complex, relating to people, events, and situations characterized by more variables than anyone can manage to identify, see in relationship, or operationalize". The simulation paradigm is equipped with tools and techniques that help researchers address these complex relations, and analyze the situations where these relationships take place.

A model is defined by Gilbert and Troitzsch (2005) as "a simplification-smaller, less detailed, less complex, or all of these together-of some other structure or system" (p. 2). They further state that through a process of abstraction, a model is built (either through a set of mathematical equations, statistical equations or a computer program) which could in turn be used to develop new theories.

For the purposes of this research, an understanding how individuals with different philosophical profiles tend to solve complex problems is going to be established through this type of simulation. Agent-Based modeling has been chosen as an appropriate tool for this research, since it possesses certain characteristics that are needed in theory exploration, as well as gaining insight on complex situations. Because computer simulations provide the capability of sharing a methodology, experimentation and data explicitly, thorough inspection and replicability is also established (Abrahamson \& Wilensky, 2005).

Gilbert and Troitzsch (2005) provide an explanation on why simulation seems to be a proper fit for social studies:

"The major reason for social scientists becoming increasingly interested in computer simulation is its potential to assist in discovery and formalization. The process of 
formalization, which involves being precise about what the theory means and making sure that it is complete and coherent, is a very valuable discipline in its own right" (p. 5)

Gilbert and Troitzsch also state that the initiation point when using simulation as a method is that there is a real world phenomenon (the target) that the researcher is interested in investigating. The main purpose of the simulation, therefore, is to create a model of that target, which would be simpler to study. However, aside from this simplification, the model also needs to be dynamic, since in social sciences the target is constantly changing over time, and reacting to its environment. Having decided on what the model should comprise, a representation of the model as a specification needs to be formulated. This specification can be made in terms of a mathematical equation, a logical statement, or a computer program. In some instances, especially when this specification is not linear, analytical reasoning may be difficult, or even impossible. That is why, Gilbert and Troitzsch conclude, simulation is often the only way.

As a result of the vast amount of research that has been done on $A B M$, it can be said that there is consensus to some degree on what characteristics an agent should possess. Taking into consideration a sample of these researches (Kauffman, 1995; Jennings, Sycara \& Woolridge, 1998; Gilbert \& Terna, 2000; Bonabeau, 2002; Macal \& North, 2005), here is how an agent can be defined:

1. Agents are identifiable, self-contained, discrete individuals, possessing their own sets of characteristics and rules. This means that an agent has its own boundaries, which helps determine whether something belongs to an agent, or is outside of the boundaries of that agent.

2. Agents are situated in an environment, which provides the medium for agents to interact with other agents. There certain rules for the interaction of agents.

3. Agents are goal-directed, meaning that each agent has a goal to achieve.

4. Agents are autonomous; they independently seek their own goals based on their own local information. There is no central authority, controller or planner. This makes selforganization possible.

5. Agents are interdependent. The actions of each agent influence the others.

6. Agents follow extremely simple rules, simplest and best supported assumptions about individual agent behavior.

7. Agents are flexible, meaning that they have the ability to learn and adapt their behaviors over time. This is reflected at the instances where an agent has a form of 
memory, and it may have some rules that modify its behavior according to these memories.

Following these and previously discussed steps, the Agent-Based model will be constructed in the following part.

\section{Research Methodology}

The main research steps start with the Concept. This is followed by the Concept to Computer (C2C) phase. In this step, the previously developed conceptual model and the premises are shaped and formalized in such a way that they can be used as input for the computer simulation model. This can be thought of as the pseudo code. Having established this, the final phase (Computer) consists of building the computer simulation model.

\subsection{Conceptual Step}

The conceptual phase consists of the previously developed, identified and selected premises, rules and the context.

\section{Premises}

1. Philosophical paradigms as underlying dimensions for personality profiles when dealing with complex problems
a) The philosophical profile of the individual (PPI) contains three philosophical dimensions; Epistemology, Ontology and Teleology.
b) Epistemological dimension contains Empiricist and Rationalist predispositions.
c) Ontological dimension contains Substantive and Process predispositions.
d) Teleological dimension contains Goal-oriented and Not Goal-oriented predispo- sitions.

2. How these profiles deal with/solve complex problems

a) What makes a problem complex?

i. Lack of information

ii. High number of entities

iii. Ambiguity

iv. Instability

v. Temporal constraints 
b) Level of complexity

c) How do the profiles deal with complex problems?

i. Each predisposition will have a different way of dealing, i.e. different capabilities.

ii. When they are combined, what happens?

\subsection{From Concept to Computer}

The Concept to Computer $(\mathrm{C} 2 \mathrm{C}$ ) process, presented below in Figure 3, involves taking the above conceptual theory, structuring it and formalizing it in a way that it can be put into a simulation medium ( $A B M)$ using a particular tool (NetLogo). An important point to address here is that the below process is nonlinear; iterations, trials and errors, and modifications are present in every step.

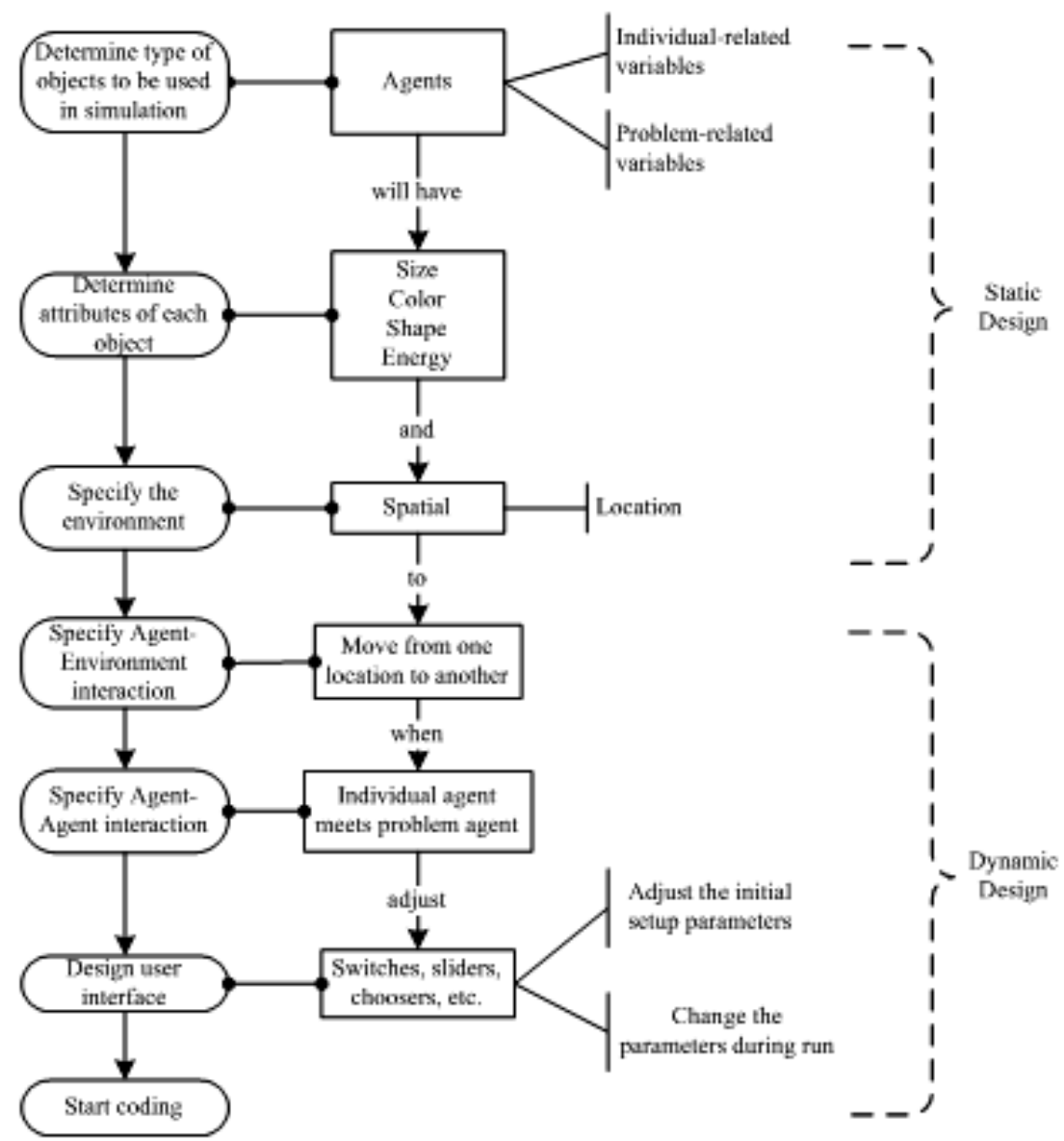

Figure 3. C2C Process (Gilbert \& Troitzsch, 2005) 


\subsection{Computer Implementation Step}

This last stage is where the agent-based model is built, consisting of two phases. Phase 1 presents an explanation on how the user-model interaction takes place, and Phase 2 is a detailed discussion on the actual simulation model, and what is taking place inside the simulation run. Therefore, these two levels occur simultaneously. The user is responsible from the initial setup conditions, and the running of the program. While the simulation is being run according to the rules and interactions described in Phase 2, the user can directly observe the outputs identified. A more detailed analysis of this output takes place afterwards.

\subsubsection{Representation of the Individual}

The three dimensions of the profile for the individual (from now on "individual agents") are represented as separate agents. The main premise of the PPI is that the personality profile is composed of three philosophical dimensions, and these dimensions have two main components which represent the predisposition of the individual. Building up on that premise, the second component of this research is to see how these predispositions, dimensions and profiles deal with problems of certain complexity. All agents possess common attributes, namely shape, size, location, number, and movement, energy and ID.

- Shape: Each of the philosophical dimensions (epistemology, ontology and teleology) is represented by a different shape.

- Size: The individual agents have a size of 1.4. This value is different from the size for the problem agents not only because of visual purposes, but mainly because of certain tracking down and output plotting purposes.

- Location: Each of the individual agents is randomly placed when created.

- Number: The initial number of individual agents is determined by the user and can be between zero and fifty, with increasing increments of 2 (i.e. 0, 2, 4, etc.). The reason for this is explained in latter sections.

- Movement: When asked to move, all problem agents move one step forward, then make a random $90^{\circ}$ turn, and move one step forward again. The purpose behind the movement is to create a means of interaction for the agents representing a problem and an individual.

- Specific variable: All individual variables have certain "energy". For the individual agents, this represents the capability of the specific predisposition to solve the specific 
problem. This capability is a randomly assigned number greater than zero, and smaller than 120.

- Epist_id/Ont_id/Tel_id: The individual agents own a specific "identification (id)". This becomes an important point in the representation of the individual agents. So far, the above explanations have been in relation to the three philosophical dimensions. Now, the focus turns to the predispositions, which is what the id represents. The Epistemological id may either be Empiricist or Rationalist. The Ontological id may either be Substantive or Process. The Teleological id can either be Goal or No Goal. Three scales, called the "predisposition scales" (Figure 4), have been created, which is controlled by a slider in the ABM. These scales represent the different predispositions, more specifically, how much of those predispositions are present within the model interface. The scale is represented as a percentage, with increasing increments of 10 .

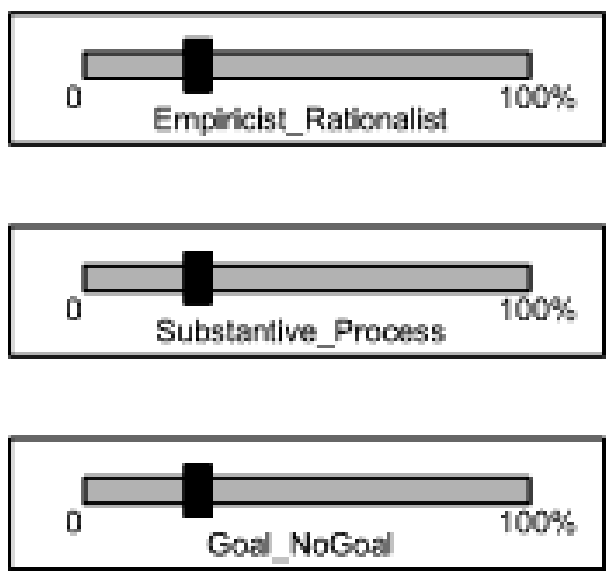

Figure 4. Predisposition Scales

The first scale, related to the Epistemological dimension, is the Empiricist/Rationalist scale. The initial value of the scale is determined by the user. The extreme ends of the scale represent the two opposite predispositions. For instance, if the percentage is set at $0 \%$, this means that all of the epistemological agents are "empiricist" agents. If the scale is set at $10 \%$, this means that $90 \%$ of the epistemological agents are empiricists, and $10 \%$ of the total epistemological agents are "rationalist" agents. If the scale is set at $100 \%$, this means that all of the epistemological agents are "rationalist" agents.

- Color: According to the specific predisposition scale determined initially by the user, the Epistemological agent will have the color "blue" or "sky"; the Ontological agent will have the color "magenta" or "violet", and the Teleological agent will have the color "brown" or "yellow". 
Figure 5 represents the steps taken to create an individual agent. As described above, when an individual agent is created, the size and location are preset in the sense that the user does not have any control over these settings. The user, however, determines how many agents there will be through the use of the "number of individual agents" slider. The user can also determine which of these agents will be representing which predispositions. In other words, if the user wants to explore PPI1 (ESG), he/she will set the Empiricist_Rationalist scale to $0 \%$, which ensures that all of the epistemological agents will be Empiricists. Taking into consideration the type of agents, the program determines the shape and the color of the agents. Each agent, as discussed above, possesses a certain capability. This capability is also set randomly. According to all of these settings, a certain number of agents are created and located randomly throughout the spatial environment; in this case, the aggregate of individual agents are now representing PPI1.

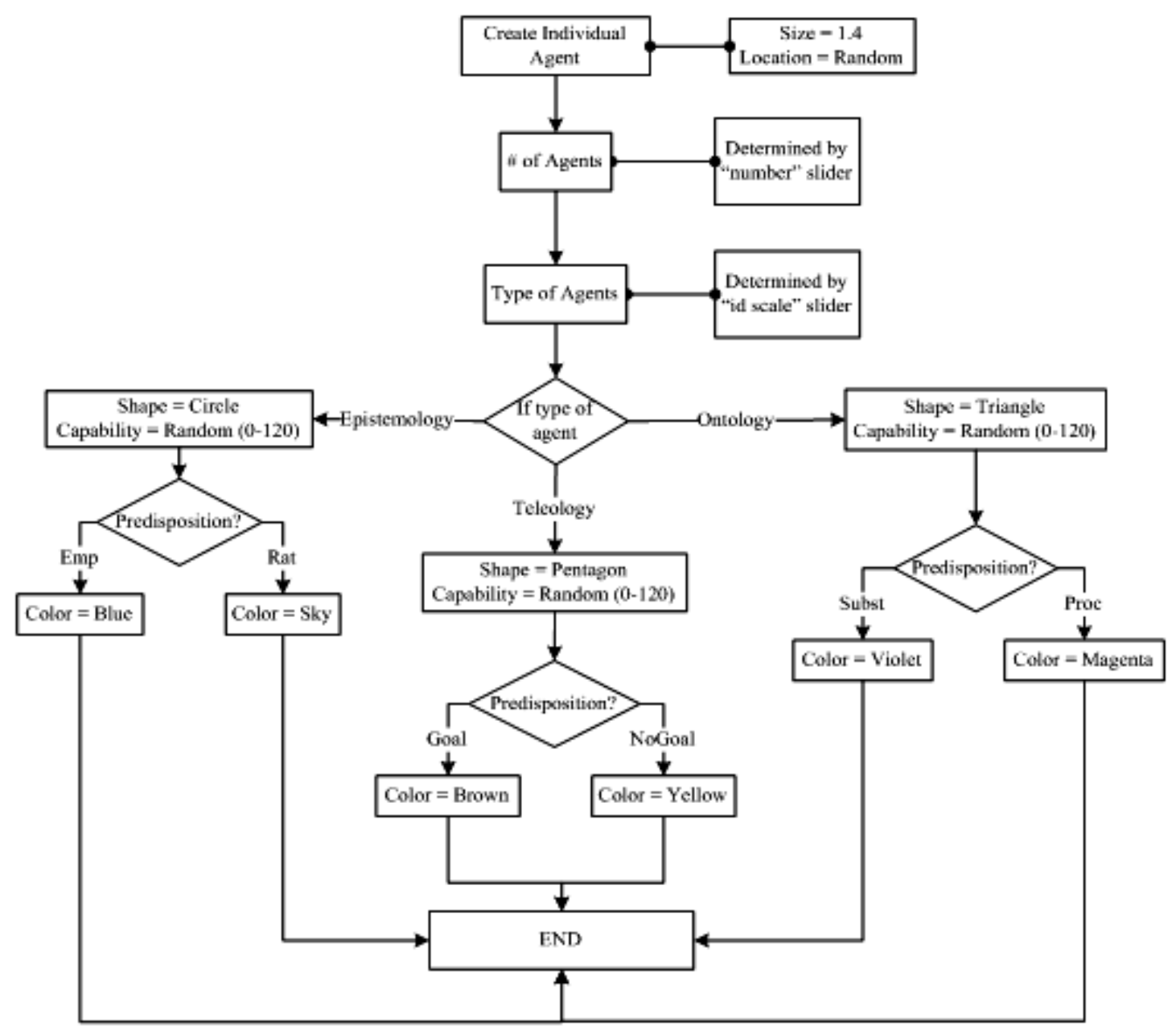

Figure 5. Setup for Individual Agents 


\subsubsection{Representation of the Complex Problem}

Each of the five variables that constitute a complex problem is represented as a different agent following the rules below:

- Shape: All problem variables (from now on "problem agents") have similar shapes, for better visualization purposes. In this case, each problem agent is represented as a different type of die.

- Size: All problem agents have a size of 1.5 .

- Location: When created, all problem agents are placed at a randomly chosen location.

- Movement: When asked to move, all problem agents move one step forward, then make a random $90^{\circ}$ turn, and move one step forward again.

- Number: The initial number of problem agents is determined by the user, and is between zero and 50 . The number of agents can be increased at increments of 10 (i.e. 10 agents, 20 agents. etc.). The reason is explained in the latter sections.

- Specific variable: All problem agents have certain "energy", called difficulty. This represents the level of complexity of the problem agent. This research uses the classification Simple-Medium-Complex. Having only Simple and Complex would be a useful simplification; however, problems do not change from complex to simple at an instant. The difficulty of a problem agent is randomly assigned when the agent is created. It can be greater than zero, and smaller than 120. The maximum limit could have been any number. The level of complexity is divided into three (simple, medium, and complex), and is reflected in the "difficulty" attribute of the problem agent as such:
a) if $>=80$ (difficulty of problem agent) < 120, problem agent: high complexity
b) if $>=40$ (difficulty of problem agent) $<80$, problem agent: medium complexity
c) if $>0$ (difficulty of problem agent) $<40$, problem agent $=$ simple

- Color: The level of complexity is reflected on the problem agent in terms of color. The "simple" problem agents are green, "medium" problem agents are orange, and "high" complex agents are red.

- Overall Problem Complexity: Even though each problem variable is represented separately, the overall problem complexity is relevant to the model, and is therefore represented as the aggregate value of individual problem variables; that is, the overall complexity of the problem agent will be equal to the sum of "information", "entity", "stability", "ambiguity", and "time" agent complexities. 
Figure 6 represents the above steps for the creation of the problem agents. When a problem agent is created, similar to the individual agents, the size and location are preset. The initial number of agents is determined through the "number of problem agent" slider. The level of complexity of the problem is determined as discussed above. The color and the difficulty values for the agents are determined according to the initial settings the user chooses.

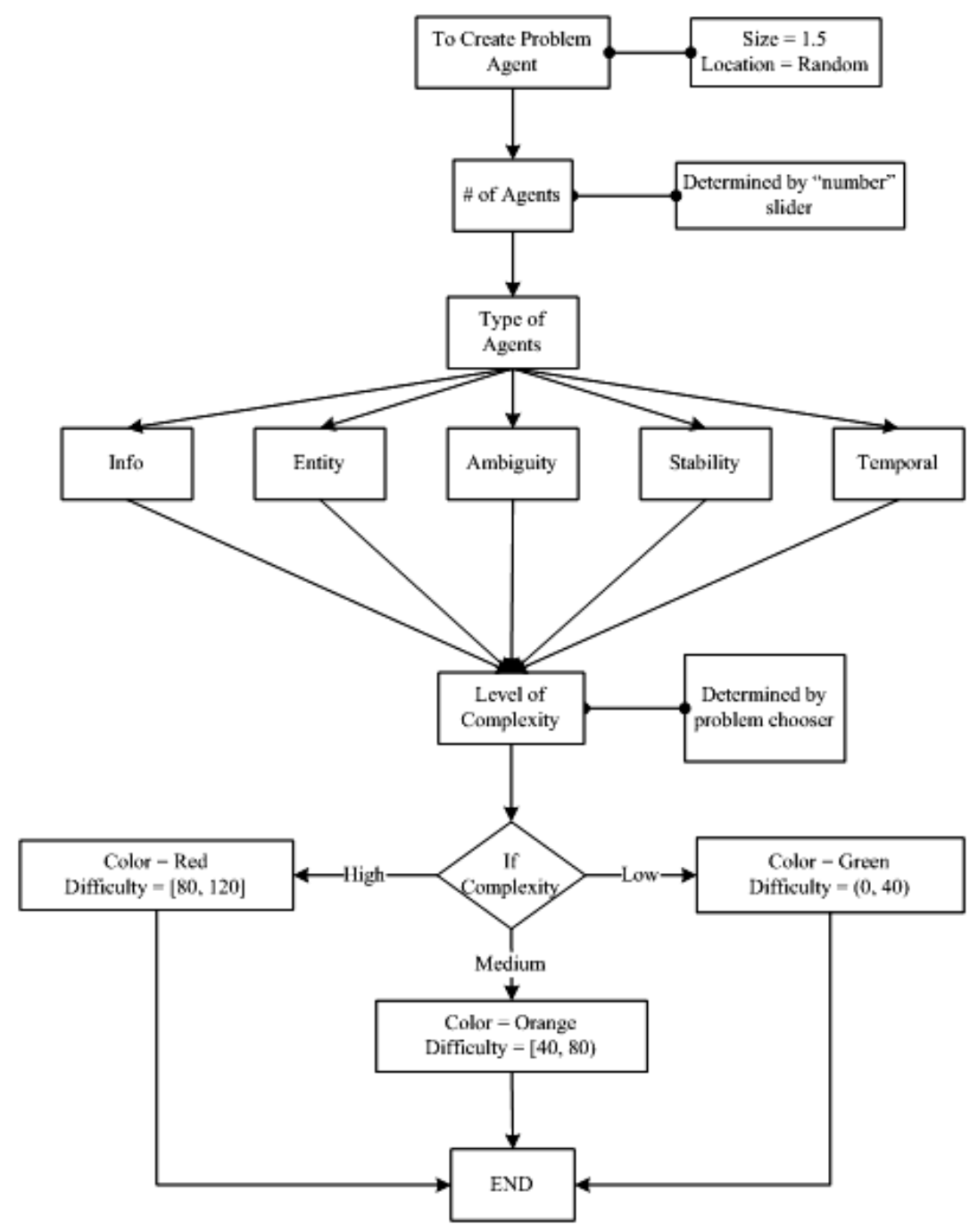

Figure 6. Setup for Problem Agents

\section{Rules}

After going through the general attributes for all agents, and specific attributes for problem and individual agents, this section provides the elaboration for the rules that the agents follow. There are three sets of rules that the simulation environment can contain: (1) Rules (characteristics) of individual agents, which are considered to be "private" information, provided to each individual agent such that they are ready to be input in the NetLogo environment, for agents to follow; (2) Rules that are common to all agents, which are 
considered as "public" information, and are accessible and available to all of the agents; and (3) Rules for connecting the agents, which is considered as coupling; where it will provide the conditions where one agent may connect with another.

\subsection{Public Rules}

Public rules are specific rules, or instructions that are given to all the agents. These rules are accessible by all the agents at any time.

$\checkmark \quad$ All agents are created

- $\quad$ size, shape, color

$\checkmark \quad$ All agents are placed

- randomly

$\checkmark \quad$ All agents move

- choose random direction, move one step

$\checkmark \quad$ All individual agents interact with all problem agents

- Capability of the individual agent will change

- Difficulty of the problem agent will change

$\checkmark$ The individual agents do not interact with each other

$\checkmark$ The problem agents do not interact with each other

$\checkmark \quad$ All agents die

- for Problem: if difficulty $<=0$, then die

- for Individual: if capability $<=0$, then die

\subsection{Private Rules}

These rules are customized for each agent, meaning that each type of agent will have a different set of private rules. They basically tell each agent what to do, and when to do it. These set of rules can be thought of as goals, or purposes that are provided to the agents, for them to achieve it, which is in line with the requirements Abrahamson and Wilensky (2005) discuss. These describe the behavior and action of the problem and individual agents. Both the individual and the problem agent are assigned a goal, as well as a criterion to establish whether that agent has met its goal or not. 


\section{Individual Agents (Purpose is to solve problems):}

$\checkmark$ look for problem variables

$\checkmark \quad$ if find a problem agent

- try to solve the problem: the capability that was assigned to the individual agent in the beginning will change.

$\checkmark$ continue moving

$\checkmark$ Criteria for individual profile: How do I know if the profile is effective?

- Success

- Consistency

\section{Problem Agents (Purpose is to be solved):}

$\checkmark$ if met with an individual variable

- try to get solved: the difficulty that was assigned to the problem agent in the beginning will change.

$\checkmark$ continue moving

$\checkmark$ Criteria for problem: How do I know problem is solved?

- Individual problem variable solutions

- Overall problem complexity

The above steps are explained in Figure 7. 


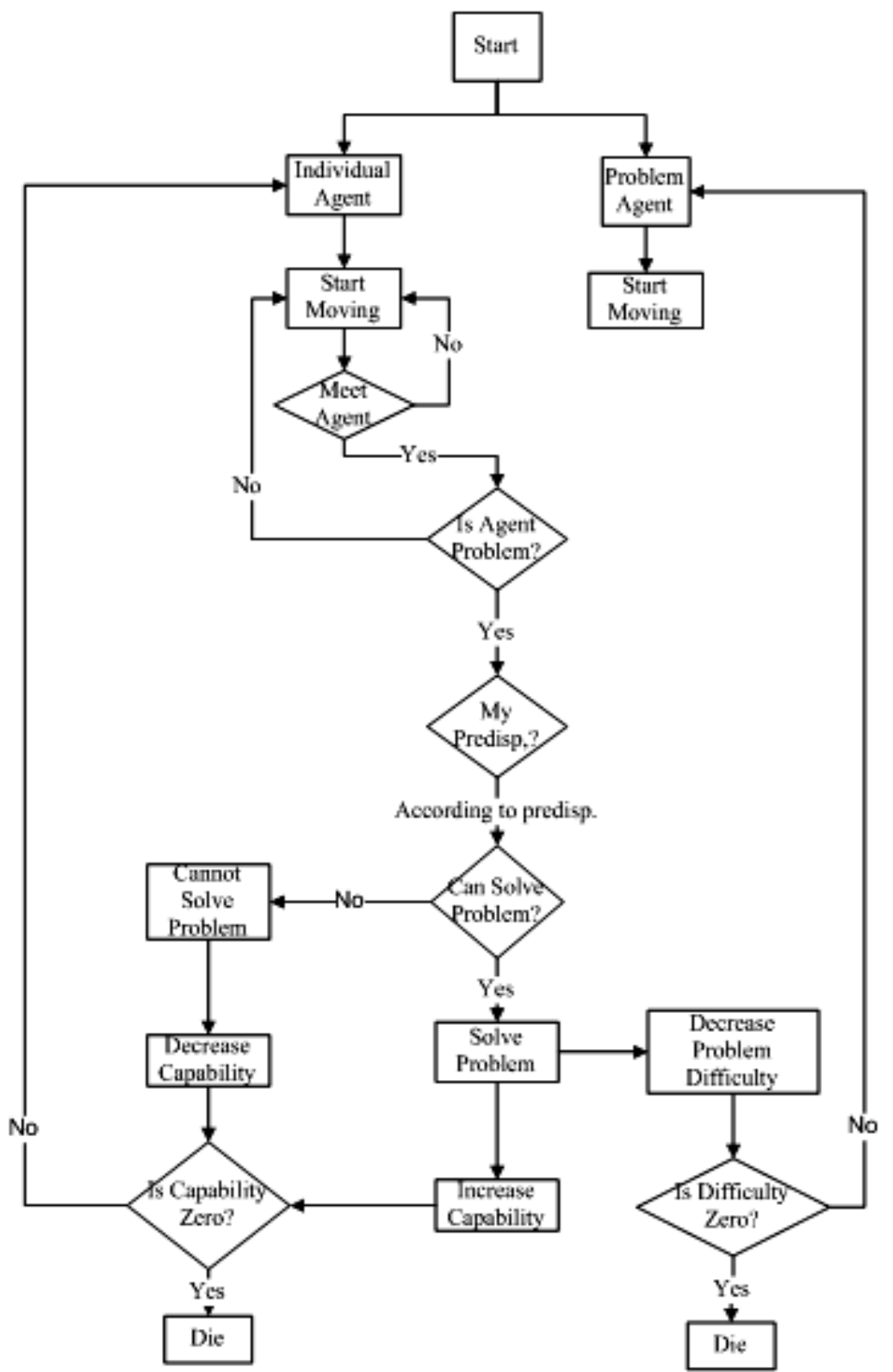

Figure 7. Common Rule Diagram for Interacting Agents

\subsection{Interaction Rules}

These rules are interaction rules for each of the individual agents with the problem agents. In below figure (Figure 8), an example of these rules is given. The figure represents what happens when an individual agent who is an empiricist meets five of the problem variables. " $C$ " denotes complex, and " $\mathrm{S}$ " denotes simple. For example, when available information about the problem is High, this individual will deal with the problem better, because the capabilities are stronger, since the tools he has makes him predisposed in a way such that he will want to collect information on the problem first. This may take a longer time (which becomes important when the problem is time-dependent), but this way makes sure that the problem is correctly identified. This is operationalized as "problem solved". When available information about the problem is Low, in other words, when the problem cannot be defined properly, the 
Empiricist will deal with the problem poorly. This is operationalized as "problem not solved". The individual will want to collect information, but will fail to do so. This will lead to an incomplete understanding of the problem. Cooper, Folta and Woo (1995), for instance, found that entrepreneurs with relevant industry experience will still perform more information search on a problem.

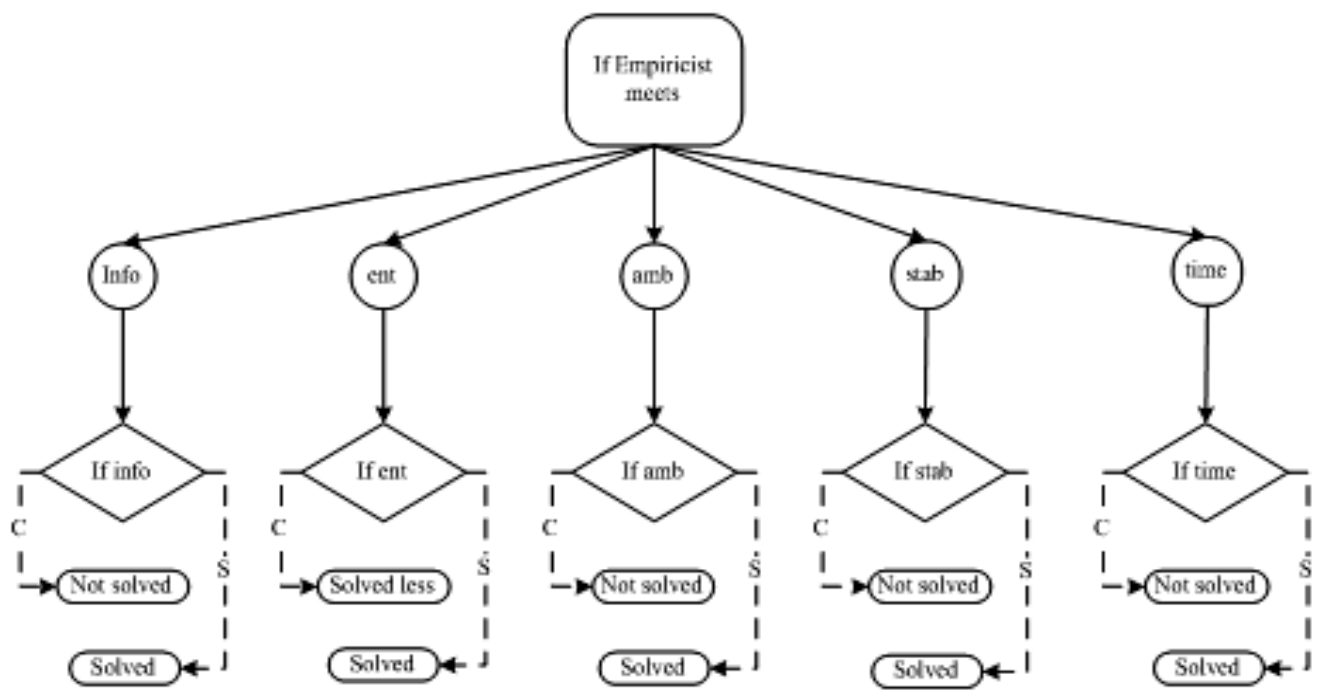

Figure 8. Sample Interaction Rules for Empiricist Agent

As seen from above figure, there are different ways a problem can be solved, or not solved. As previously discussed, there are two variables that change during these interactions: the problem difficulty and the predisposition capability. The following rules are used for these changes in the variables (Table 1).

\begin{tabular}{|l|c|c|}
\hline & Capability & Difficulty \\
\hline Problem not solved & -2 & No change \\
\hline Problem not solved less & -1 & No change \\
\hline Problem solved & +2 & -2 \\
\hline Problem solved less & +1 & -1 \\
\hline
\end{tabular}

Table 1. Rules for Capability and Difficulty

- Problem Not Solved: This rule indicates that the predisposition did not have enough capabilities to deal with that particular variable, therefore lost capabilities with an increment of -2 . The problem difficulty does not change.

- Problem Not Solved Less: This rule is a modification on the first one. The profile may still not have enough capabilities to deal with a problem variable, however in a slightly 
lesser degree. For instance, the disadvantage an Empiricist has over lack of available information is not in the same extent that a Goal-oriented individual has with an ambiguous problem, hence the decrease in capability with an increment of -1 . However, the problem difficulty still remains unchanged.

- Problem Solved: This rule indicates that the predisposition does have strong capabilities when dealing with that particular problem variable, e.g. an Empiricist agent facing an information variable with a high level of available information. In this case, this agent is able to solve that particular problem variable, and in turn increases its capabilities by +2 . The problem difficulty decreases by -2 .

- Problem Solved Less: This rule, similar to the second one, is a modified version of the previous rule. In this case, the predisposition is still capable of dealing with a particular problem agent, albeit in a lesser degree. Therefore the capability gain is +1 , and the problem difficulty is decreased by -1 .

These subtle differences are necessary in order to capture the nuances within predispositions, and the way they deal with each problem variable. Without these different rules, the emergent capability of the agent-based model would have been ignored, and the whole purpose of using this specific method would have been defeated.

The steps of how the computer simulation is going to take place are summarized in Figure 9.

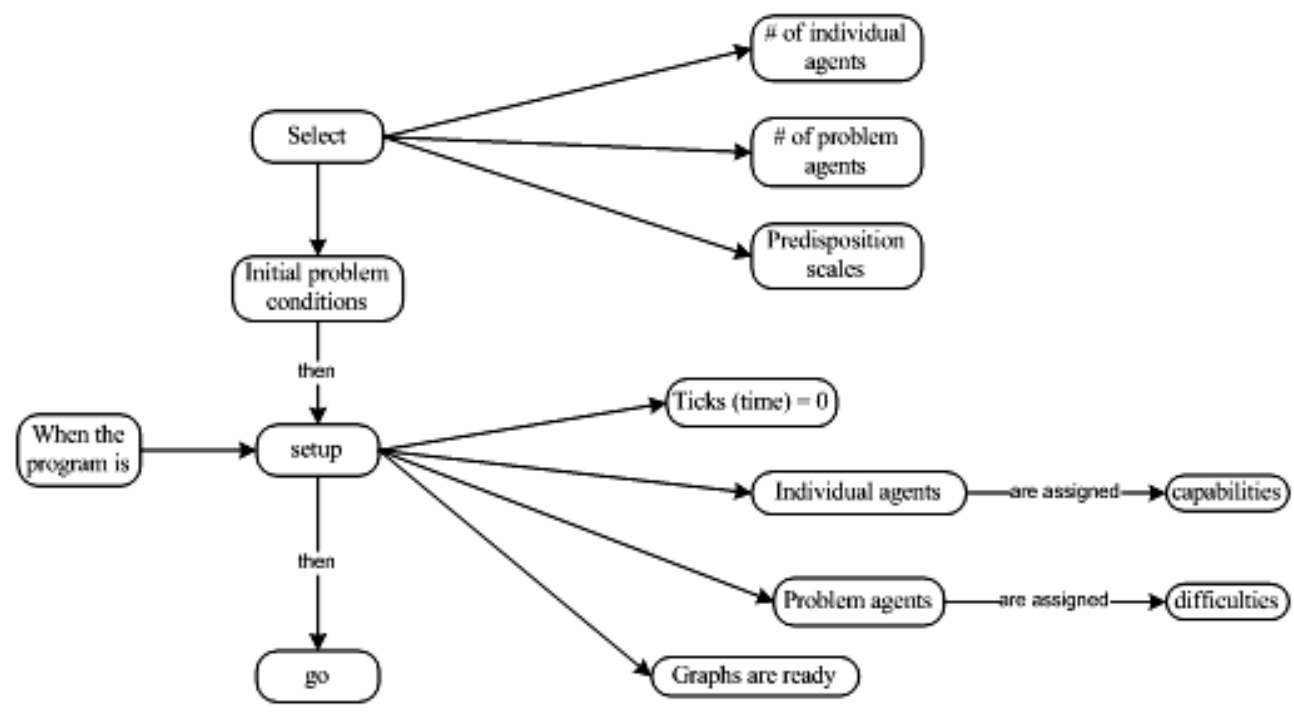

Figure 9. Overall Rule Chart

As the first step, the user selects the number of agents describing the problem variables, and the number of agents describing individual predispositions. Following this, through the predisposition scales, the user selects the profile to be explored. The problem conditions can 
be determined by the user as well. Each of the five variables (available information, number of entities, stability, ambiguity and time) can be adjusted separately. Time for the simulation run can also be adjusted according to the needs of the user. The run can have no time limit; in this case the model is coded in such a way that it would terminate itself when there are no individual or problem agents. When the user hits the "setup" button, the predetermined number of agents will be created and randomly distributed on the interface, which is the environment in which the agents interact with each other. The individual agents are assigned certain capabilities, and the problem agents are assigned difficulties.

When the program is started with the "go" button, both the individual and problem agents will start moving randomly. This means that there is now a problem that the individual needs to address. The individual agents look for problem agents to solve. Once the individual agent comes across a problem agent, the individual agent determines what type (e.g. "info") and what level (e.g. "high") that problem agent is. According to the characteristics of the problem agent and the individual agent itself, the individual will either solve the problem or not. If the individual agent can solve the problem, the capability of that agent will increase, and the difficulty of the problem agent will decrease. If the individual agent cannot solve the problem, the capability will increase, and the problem difficulty will remain the same. This process continues until there are no more problem or individual agents remaining.

\subsection{Evaluation Criteria}

In order to accurately determine and interpret the results, certain criteria need to be established. The first is related to the individual, and the second is related to the complex problem.

\section{Criteria for the Individual:}

This is related with the effectiveness of the PPI under question, and tries to answer the question: How do I know if the profile is effective?

- Success: The better an individual agent is with a problem agent (according to the rules above), the more capabilities it will gain when solving the problem. Therefore, when an individual agent attempts to solve a problem agent that it is comfortable solving, the capability of the individual agent will increase. If not, the capability of the individual agent will decrease.

- Consistency: This will depend on how successful an individual agent is over time, in different runs. It is crucial to look at whether or not the capability of the particular 
predisposition, as well as the overall capability of the profile increases or decreases in a similar fashion when different runs under same conditions are tried.

\section{Criteria for the Problem:}

- This is related to the problem that the individual is attempting to solve, and tries to answer the question: How do I know problem is solved? The problem is considered solved when the difficulty is equal to zero. The difficulty of a problem agent will decrease if the individual agent is comfortable with dealing with that type of problem. If not, the difficulty will increase.

Figure 10 below is a snapshot of the NetLogo agent-based model that was constructed as discussed above.

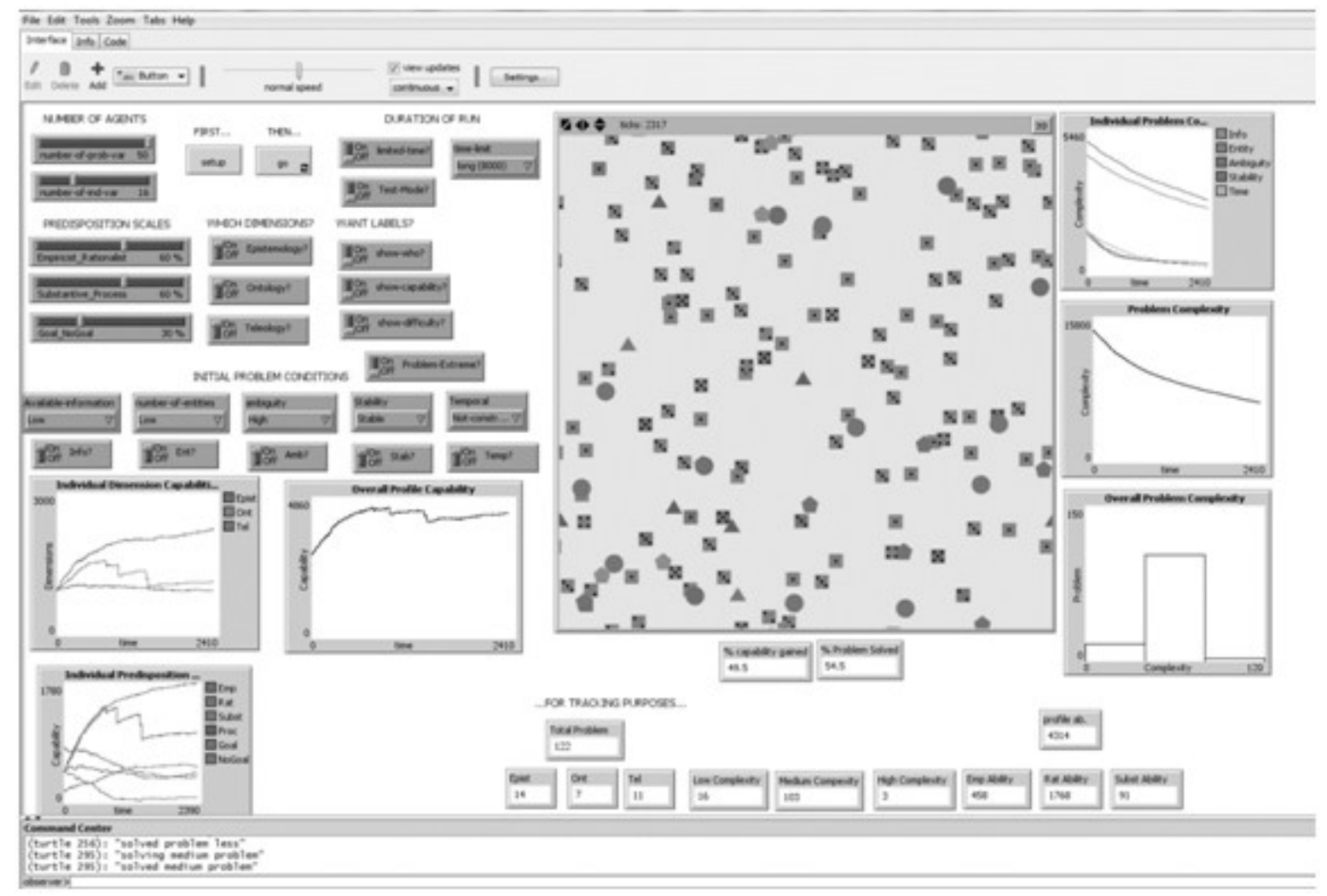

Figure 10. Snapshot of the PPI Model

\section{Results and Discussions}

In this section, specific results achieved as part of the simulation will be presented, as well as discussions on the significance of these results. The second part of this section includes discussions on the canons of research, such as verification and validity. 


\subsection{Simulation Results}

In order to compare all eight profiles with each other, two batches of analyses were conducted. The first analysis is conducted on the problem solved, and the time it took the profiles to solve the complex problem. The second analysis is on the capabilities of the profiles. The bar chart below was obtained from the individual results of the simulation runs. Figure 11 is a representation of the time it took for each profile to reach $80 \%, 90 \%$ and $100 \%$ solution for the complex problem. Within the model, the complex problem is solved completely by each of the profiles. However, the time it takes for the profiles to do this is different, depending on the predispositions of the profiles.

According to results shown below, PPI8 (RPN) solved a complex problem completely in the least amount of time. This is followed by PPI7 (RSN). In both these cases, the ontological predisposition was Rationalist, and the Teleological predisposition was NoGoal orientation. When dealing with a complex problem, the results show that these particular predispositions provide useful capabilities to the individual. On the other hand, the profiles that needed the longest time were PPI1 (ESG) and PPI4 (RPG). The goal-orientation of the profile works as a disadvantage in the case of these two profiles.

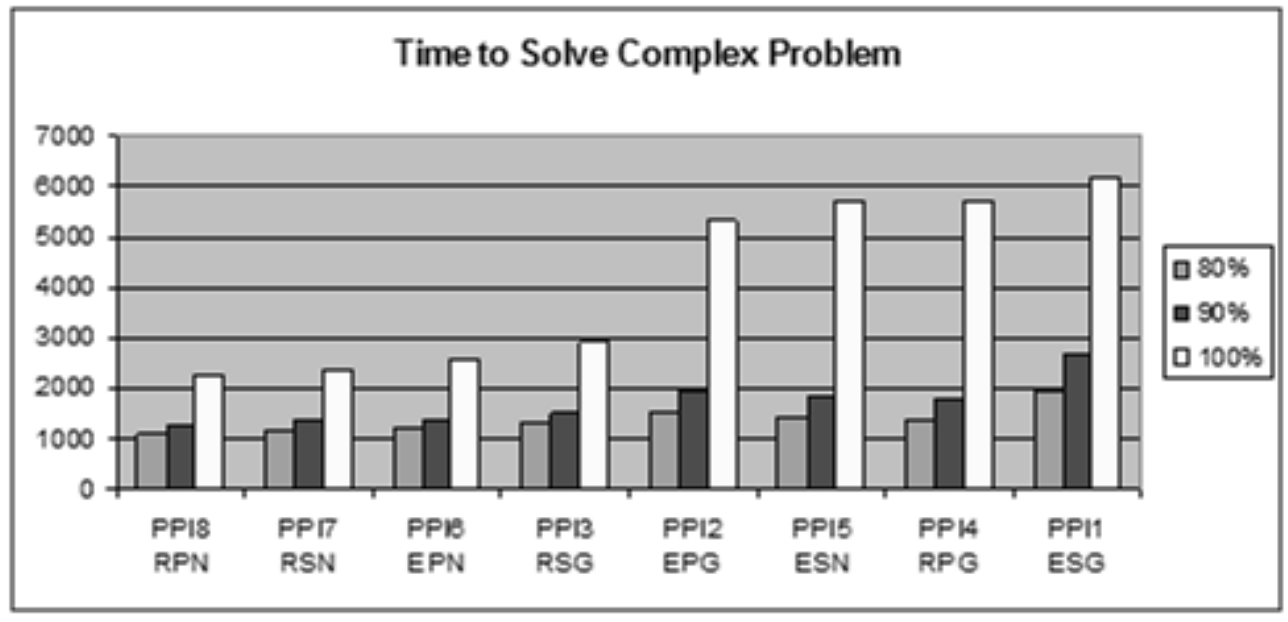

Figure 11. Comparison of Profiles for Complex Problem Solution

Some interesting conclusions can be drawn from this. It could be observed that the profiles were clustered into 2 groups; the first group consisting of PPI8, PPI7, PPI6 and PPI3; and the second group being PPI2, PPI5, PPI4 and PPI1. With the exception of PPI3 and PPI5, the two groups are divided according to their teleological dimension, with the profiles having no goal orientation on the left hand side, indicating quicker problem solution, and the goal oriented profiles taking a longer time to solve a problem. Even though PPI5 (ESN) shares the same ontological and teleological predispositions with profile PPI7 (RSN), the epistemological predisposition is the dominant one in this case. 
In order to conduct a deeper analysis of this, the following ANOVA table has been obtained for the eight profiles (Table 2).

\begin{tabular}{|l|c|c|c|c|c|}
\hline \multicolumn{7}{|c|}{ ANOVA } \\
\hline & Sum of squares & df & Mean square & F & Sig. \\
\hline Between groups & 965335.396 & 7 & 137905.057 & 180.157 & .000 \\
\hline Within groups & $2.64 \mathrm{E}+07$ & 34440 & 765.473 & & \\
\hline Total & $2.73 \mathrm{E}+07$ & 34447 & & & \\
\hline
\end{tabular}

Table 2. ANOVA for all profiles solving problems

As seen from above table, the eight groups are different from each other $(p<0.05)$. However, in order to address the differences between the profiles more accurately in terms of time, further analysis have been conducted for the profiles considering the similarities according to the previous bar chart.

The traditional post hoc analysis methods such as Tukey's could not be used in this case, because the samples did not pass the homogeneity of variances test. As a result of this, Games-Howell post hoc analysis was conducted. The results obtained showed that, similar to the bar chart above, PPI2, PPI4 and PPI5 were not significantly different from each other and therefore formed one cluster. The same results were seen for PPI6, PPI7 and PPI8, which were also not significantly different from each other. However, PPI1 and PPI3 were significantly different from all of the other profiles, and from each other. These results represent the similarities and differences between the eight profiles in terms of the time it took them to solve the complex problem. The following conclusions can be drawn from these results: When the epistemological predisposition is rationalist, and there is no goal orientation, then the ontological predisposition does not have a significant effect on the overall behavior of the profile, hence the lack of difference between PPI7 and PPI8. This may be due to the fact that since the rationalist predisposition will use skills such as reasoning, deduction and logic to gain information and acquire knowledge about a complex problem, how that problem is defined (whether as individual entities, or as a sequence) does not become relevant. If the epistemological predisposition was empiricist, this would have been different, as in the case of PPI5 (ESN) and PPI6 (EPN). These two profiles are different from each other, meaning that the ontological predisposition in this case has a significant effect, since the empiricist individual will need to collect hard data and facts to understand a problem. The definition of that problem becomes relevant in this type of situation.

It can be seen that PPI3 and PPI2 are significantly different from each other as well. Even though they are different in terms of both their epistemological and ontological predispositions, one of the main premises was that the empiricist predisposition was shown to have much less 
capability when dealing with complex problems, hence the big increase in time. As seen from Figure 12, some profiles gained capability, and some profiles lost capability. This is aligned with the proposition that certain predispositions would provide better capabilities to the individual than other.

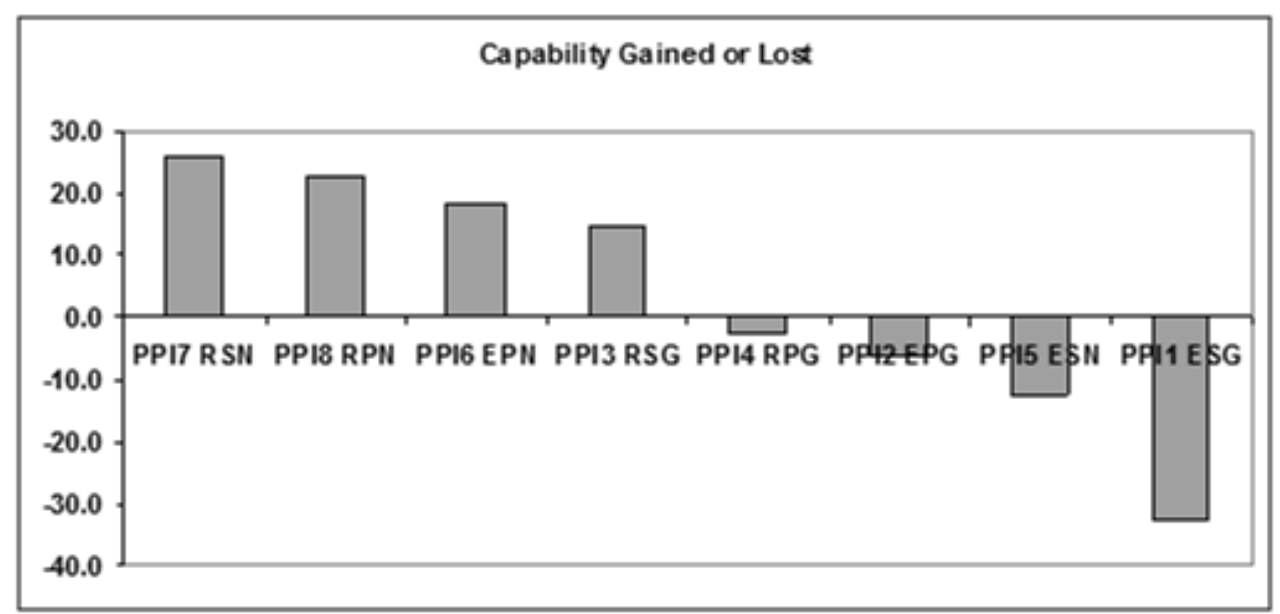

Figure 12. Comparison of Profiles for Capability Gained/Lost

Here are some initial conclusions that can be drawn from the above figure. Similar to previous results, PPI7 was the most successful in terms of gaining capabilities, followed by PPI8. PPI1 and PPI5, on the other hand, was the least successful of the eight profiles. These two profiles on the lower end of the graph contain the same epistemological and ontological predisposition, namely Empiricism and Substantive-orientation. Therefore, despite the change in the teleological predisposition, the epistemological and ontological predispositions were too dominant in terms of their disadvantages. Even though PPI7, which gained most capability, also has a substance-oriented ontological predisposition, the combination of the Rationalist and NoGoal orientations seem to provide better capabilities to the profile, as opposed to PPI5, which shares the same ontological and teleological predisposition. Similar to the results for problems solved, an ANOVA was conducted to look at all of the eight profiles in terms of their capabilities. The following table is obtained (Table 3 ). The results show that all profiles are significantly different from each other.

\begin{tabular}{|l|c|c|c|c|c|}
\hline \multicolumn{7}{|c|}{ ANOVA } \\
\hline & Sum of squares & df & Mean square & F & Sig. \\
\hline Between groups & $7.71 \mathrm{E}+10$ & 7 & $1.10 \mathrm{E}+10$ & 41028.581 & .000 \\
\hline Within groups & $9.26 \mathrm{E}+09$ & 34487 & 268403.859 & & \\
\hline Total & $8.63 \mathrm{E}+10$ & 34494 & & & \\
\hline
\end{tabular}

Table 3. ANOVA for all profile capabilities 
However, in order to look at further detailed interpretations, a post hoc analysis was also conducted. The capability variables also did not pass the homogeneity of variance test, therefore similar to the previous section, Games-Howell post hoc analysis was conducted. The results show that only PPI7 and PPI8 are not significantly different from each other. The following conclusions can be drawn from this: Similar to the problem solved, the ontological dimension did not have any effect on the capability gained by the profiles PPI7 and PPI8. In terms of capabilities gained or lost, unlike the problem solved, the profiles behaved different from each other (with the exception of PPI7 and PPI8). This means that all three philosophical dimensions had something different to contribute to the behavior of the overall profile.

\subsection{Canons of Research}

Generalizability, applicability, consistency, reproducibility, precision, verification and validation are examples of canons that a research, through its research design, must provide sufficient depth. Any research that deals with social issues, that has a purpose of gaining insight on complex problems, and that has little or no empirical foundation or opportunity of observation of phenomena under question needs to establish the appropriate canons to be used, and follow them.

External validity is related to the generalizability of the study, e.g. from a sample to a population, which is based on establishing the domain of a study. Kelijnen (1999: page 648) notes that "[i]f no data on the real system are available, then strong validation claims are impossible". From the rationalist perspective, external validity is focused on the applicability to the context from which the premises were derived. In other words, the results should make sense when being considered from various bodies of knowledge that provide the basis for the background research. In the case for this research, for instance, do the insights that have been gained make sense? Does the way an individual with a certain PPI solves complex problems make sense? For simulations in the social sciences, Kuppers and Lenhard (2005) argue that validity (in the classical, traditional sense) is not an adequate measure. As Bossel (1994) states, the construction of a model will always include simplifications, aggregations, omissions and abstractions. Sargent (1999) stated that developing a model should be based on a specific purpose, and the validity of that model needs to be considered with respect to that purpose. Bossel (1994) had argued the same point when he stated that a simulation model cannot be discussed in terms of its correctness but only in terms of its validity with respect to the model purpose.

Construct validity is defined as "the degree to which inferences can legitimately be made from the operationalizations in [the] study to the theoretical constructs on which those operationalizations were based" (Trochim, 2006). In other words, establishing construct validity makes sure that correct operational measures are used for the concepts that are being 
studied (Yin, 2003). When considered with respect to the rationalist deductive methodology, this type of validity is applicable during the structuration \& development stage. Construct validity is relevant to the premises being made explicit. Similar to internal validity, construct validity is based on the coherent structure of the premises and the way they are formulated in order to describe and build knowledge claims. The way the premises can be traced back to literature and past theories is an indicator for this type of validity.

Balci (1997: page 1) defines verification of a model as "substantiating that the model is transformed from one form into another, as intended, with sufficient accuracy". In other terms, it makes sure that the model is built right. According to Sargent's definition (Sargent, 1999), verification of a model ensures that the computer program of the model and the implementations are running correctly. According to Sargent (1999), is a simulation language is used within the computer programming, model verification makes sure that the simulation language is error free, has been properly implemented, and has been correctly programmed. Some researchers, the verification phase is identical to the internal validity phase and includes debugging, which is done to ensure that the computer program is running as intended. There are certain steps that need to be taken in order to verify the simulation model. The following steps are described by Gilbert (2008), as well as Macal and North (2007):

- Code elegantly: This advice ties back to the core of modeling and simulation, where the coding needs to be as simple as possible, but not too simple that necessary details could be omitted. It was the intention of the author to use as simple and few lines as possible, while making sure that all the details of the model were incorporated within the implementation.

- Include output and diagnostics: NetLogo provides a range of buttons, sliders and monitors to trace each step of the simulation, and to ensure that each output and outcome from the simulation is somehow captured through the many tools available to the modeler.

- Observe simulation, step by step: This step is tied together with the previous one. Setting up the correct trackers and monitors gives the user the advantage to observe what exactly is going on at any point of the simulation. Slowing down the simulation process also is a useful approach that was adapted by the author. The movement of each agent and their interactions through a simulation run one time step at a time was observed. This way, any anomalies that occur during the simulation could be caught as soon as they occur.

- Add comments and update them: The Command Center feature that is available in NetLogo can be, and was, used to track down every movement that occurred in the simulation. For instance, when an agent representing an individual cannot solve a 
particular problem, the Command Center reads "couldn't solve complex problem", and according to the rule assigned to that particular agent, the capability of the agent should decrease. Another example would be "could solve medium problem", where the capability is expected to increase. By reading this, and also looking at the agents, the researcher could see if what was happening was actually a match with what was coded.

- Use unit testing: In this research, this is done by separating the NetLogo program into sub-sections of code, transferring those particular lines of code into a separate file and making sure that each batch of code runs without any error.

\section{Conclusion}

This research does not attempt to develop a fully comprehensive and detailed model of how individuals think, behave and act. Such complexity is almost impossible to understand in real life, let alone in a simulated model. However, what this research provides is a means to gain insight on a small portion of individual personalities, on how individuals with different philosophical profiles deal with complex problems. It has been established by many different studies (both academy and industry-oriented) that the application of personality profiles increase the productivity of the firm, as well as the productivity and satisfaction of the individual. Self-awareness is an extremely useful and necessary concept to have within an individual's life. If we are aware of our predispositions, our worldviews, our inner world, we will function better in the outer world. Establishing philosophical profiles of individuals will also prove to be useful when it comes to interaction with other people, building teams etc. It is clear that individuals with certain predispositions will work better with individuals who possess the same worldviews. Agent-Based modeling has been chosen as an appropriate tool for this research, since it possesses certain characteristics that are needed in theory exploration, as well as gaining insight on complex situations.

The conceptual, methodological and practical implications of the research provide various opportunities for future research topics. One such future research area is transferring the research to an empiricist paradigm and empirically analyzing the profiles that have been developed, proposed and explored. The profiles here presented are done so in a rationalist manner, through the use of a computer simulation, and an assessment on how to empirically test the PPI is part of a work in progress. The initial step taken for this is the operationalization of the profiles. This is done by developing a questionnaire (PPI-Q) in order to test and validate the dimensions, as well as the profiles themselves. Statements such as "In order to accept something to be true, I would need to have actual proof" and "I like to be organized and plan ahead" are used to determine the individual's predispositions and relevant profiles. Through appropriate demographic studies, profiles of different groups such as academics vs. practitioners can be determined. Furthermore, profiles of individuals who are in different 
professions (such as engineers, managers, leaders, scientists, etc.) can be explored. For instance, the PPI could be applied to leaders and different leadership styles. This future research may focus on capturing the underlying philosophical assumptions of leaders and leadership. Since leadership is a topic that has been researched ad nauseam with no common agreement within leadership scholars on what leadership theories are significant, and which ones present a holistic picture, using the philosophical dimensions of the PPI would fill a significant gap in the body of knowledge, as well as solidifying the applicability of the developed profiles. It is hoped that the PPI-Q could be used in different contexts. Having a tested and reliable tool to assess the philosophical profile of an individual certainly would have a significant impact on many overlapping bodies of knowledge.

\section{References}

Abrahamson, D., \& Wilensky, U. (2005). Piaget? Vygotsky? I'm game!: Agent-based modeling for psychology research. Jean Piaget Society Meeting, Canada.

Augier, M., Shariq, S.Z., \& Vendele, M.T. (2001). Understanding context: its emergence, transformation and role in tacit knowledge sharing. Journal of Knowledge Management, 5(2), 125-137. http://dx.doi.org/10.1108/13673270110393176

Balci, O. (1997). Verification, validation and accreditation of simulation models. Proceedings of the 1997 Winter Simulation Conference. S. Andradottir, K. J. Healy, D. H. Withers and B. L. Nelson (Eds.). 135-141.

Bandura, A. (2001). Social cognitive theory: An agentic perspective. Annual Review of Psychology, 52, 1-26. http://dx.doi.org/10.1146/annurev.psych.52.1.1

Bar-Yam, Y. (1993). Dynamics of complex systems. Westview Press.

Bonabeau, E. (2002). Agent-based modeling: Methods and techniques for simulating human systems. Proceedings of the National Academy of the Sciences, 99(3), 7280-7287. http://dx.doi.org/10.1073/pnas.082080899

Bossel, H. (1994). Modeling and Simulation. A.K. Peters, Ltd. http://dx.doi.org/10.1007/978-3-66310822-1

Bozkurt, I., Padilla, J.J., \& Sousa-Poza, A.A. (2007). Philosophical Profile of the Individual. IEEE Engineering Management Conference, Austin, TX, pp. 42-48.

Cooper, A.C., Folta, T.B., \& Woo. C. (1995). Entrepreneurial information search. Journal of Business Venturing, 10, 107-120. http://dx.doi.org/10.1016/0883-9026(94)00022-M 
Davis, J.H. (1969). Individual-group problem solving, subject preference and problem type. Journal of Personality and Social Psychology, 13(4), 362-374. http://dx.doi.org/10.1037/h0028378

Duncan, R.B. (1972). Characteristics of organizational environments and perceived environmental uncertainty. Administrative Science Quarterly, 17, 313-327. http://dx.doi.org/10.2307/2392145

Egges, A., Kshirsagar, S., \& Magnenat-Thalmann, N. (2003). A model for personality and emotion simulation. Knowledge-Based Intelligent Information \& Engineering Systems, 453-461.

Epstein, J.M. (1999). Agent-based computational models and generative social science. Complexity, 4(5), 41-60.

http://dx.doi.org/10.1002/(SICI)1099-0526(199905/06)4:5<41::AID-CPLX9>3.0.CO;2-F

Flood, R.L., \& Carson, E.R. (1993). Dealing with Complexity: An Introduction to the Theory and Application of Systems Science. New York: Plenum Press. http://dx.doi.org/10.1007/978-14757-2235-2

Funke, J. (1991). Solving complex problems: Exploration and Control of Complex Systems. In R.J. Sternberg and P.A. Frensch (eds.). Complex Problem Solving: Principles and Mechanisms. Mahwah, NJ: Erlbaum.

Ghasem-Aghaee, N., \& Oren, T.I. (2007). Cognitive complexity and dynamic personality in agent simulation. Computers in Human Behavior, 23, 2983-2997.

http://dx.doi.org/10.1016/j.chb.2006.08.012

Gigliotta, O., Miglino, O., \& Parisi, D. (2007). Groups of Agents with a Leader'. Journal of Artificial Societies and Social Simulation, 10(4), 1. http://jasss.soc.surrey.ac.uk/10/4/1.html. Retrieved on $10 / 07 / 2014$.

Gilbert (2008). Researching social life. 3rd Ed., London: Sage.

Gilbert, N, \& Abbot, A. (2005). Introduction to special issue: social science computation. American Journal of Sociology, 110(4), 859-863. http://dx.doi.org/10.1086/430413

Gilbert, N., \& Terna, P. (2000). How to build and use agent-based models in social science. Mind and Society, 1(1), 57-72. http://dx.doi.org/10.1007/BF02512229

Gilbert, N., \& Troitzsch, K.G. (2005) Simulation for the social scientist. 2nd edition. Buckingham: Open University Press.

Gratch, J., \& Marsella, S. (2005). Evaluating a computational model of emotion. Autonomous Agents and Multi-Agent Systems, 11, 23-43. http://dx.doi.org/10.1007/s10458-005-1081-1 
Holland, J. (1995). Hidden order: How adaptation builds complexity. Cambridge, MA: Perseus.

Hood, J.N., Logsdon, J.M., \& Thompson, J.K. (1993). Collaboration for social problem-solving: A process model. Business and Society, 32(1), 1-17. http://dx.doi.org/10.1177/000765039303200103

Jennings, N.R., Sycara, K., \& Woolridge, M. (1998). A roadmap of agent research and development. Autonomous Agents and Multi-Agent Systems, 1, 7-38.

http://dx.doi.org/10.1023/A:1010090405266

Kauffman, S. (1995). At home in the universe. NY: Oxford.

Kleijnen, J.P.C. (1999). Validation of models: Statistical techniques and data availability. P.A. Farrington, H.B. Nembhard, D.T. Sturrock \& G.W. Evans (Eds.). Proceedings of the 1999 Winter Simulation Conference, 647-654.

Kikuchi, T., \& Nakamori, Y. (2007). Agent model analysis to explore effects of interaction and environment on individual performance. Journal of Systems Science and Complexity, 20(1), 1-17. http://dx.doi.org/10.1007/s11424-007-9000-y

Kuppers, G., \& Lenhard, J. (2005). Validation of simulation: Patterns in the social and natural sciences. Journal of Artificial Societies and Social Simulation, 8(4), 1-13.

Lawrence, P.R., \& Lorsch, J.W. (1967) Organization and environment. Boston. Harvard University, Graduate School of Business Administration, Division of Research.

Lloyd, I. (1978). Don't define the problem. Public Administration Review, 38(3), 283-286. http://dx.doi.org/10.2307/975684

Macal, C.M., \& North, M.J. (2005). Tutorial on agent-based modeling and simulation. M.E. Kuhl, N.M. Steiger, F.B. Armstrong \& J.A. Joines (Eds). Proceedings of the 2005 Winter Simulation Conference, 2-15. http://dx.doi.org/10.1109/WSC.2005.1574234

Macal, C.M., \& North, M.J. (2007). Agent-based Modeling and Simulation: Desktop ABMS. Proceedings of the 2007 Winter Simulation Conference. Eds. S. G. Henderson, B. Biller, M.-H. Hsieh, J. Shortle, J. D. Tew, and R. R. Barton, 95-106. Washington, DC. http://dx.doi.org/10.1109/WSC.2007.4419592

Martinez-Miranda, J., \& Aldea, A. (2005). Emotions in human and artificial intelligence. Computers in Human Behavior, 21, 323-341. http://dx.doi.org/10.1016/j.chb.2004.02.010

Mumford, E. (1998). Problems, knowledge, solutions: Solving complex problems. Journal of Strategic Information Systems, 7, 255-269. http://dx.doi.org/10.1016/S0963-8687(99)00003-7 
Overwalle, F.V., \& Heylighen, F. (2006). Talking nets: A multiagent connectionist approach to communication and trust between individuals. Psychological Review, 113(3), 606-627. http://dx.doi.org/10.1037/0033-295X.113.3.606

Peshkin, A. (1993). The goodness of qualitative research. Educational Researcher, 22(2), 23-29. http://dx.doi.org/10.3102/0013189x022002023

Quesada, J., Kintsch, W., \& Gomez, E. (2005). Complex problem-solving: A field in search of a definition? Theoretical issues in ergonomics science, 6(1), 5-33.

http://dx.doi.org/10.1080/14639220512331311553

Rittel, H.W.J. \& Webber, M.M. (1973). Dilemmas in a general theory of planning. Policy Sciences, 4, 155-169. http://dx.doi.org/10.1007/BF01405730

Sallach, D., \& Macal, C. (2001). The simulation of social agents: An introduction. Social Science Computer Review, 19(3), 245-248. http://dx.doi.org/10.1177/089443930101900301

Sargent, R.G. (1999). Validation and verification of simulation models. P.A. Farrington, H.B. Nembhard, D.T. Sturrock \& G.W. Evans (Eds.). Proceedings of the 1999 Winter Simulation Conference, 39-48.

Simon, H.A. (1972). Theories of Bounded Rationality. Chapter 8 in C.B. McGuire \& R. Radner, (Eds.). Decision and Organization. Amsterdam: North-Holland Publishing Company.

Silverman, B.G., Bharathy, G.K., \& Nye, B. (2007). Profiling is politically 'Correct': Agent-based modeling of ethno-political conflict. Interservice/Industry Training, Simulation and Education Conference.

Smith, E.R., \& Conrey, F.R. (2007) Agent-Based Modeling: A New Approach for Theory Building in Social Psychology. Personality and Social Psychology Review, 11(1), 87-104. http://dx.doi.org/10.1177/1088868306294789

Steinberg, E.R. (1983). Problem complexity and the transfer of strategies in computerpresented problems. American Educational Research Journal, 20(1), 13-28.

http://dx.doi.org/10.3102/00028312020001013

Swinth, R.L. (1971). Organizational joint problem-solving. Management Science, 18(2), 68-79. http://dx.doi.org/10.1287/mnsc.18.2.B68

Sun, R., \& Naveh, I. (2004). Simulating Organizational Decision-Making Using a Cognitively Realistic Agent Model. Journal of Artificial Societies and Social Simulation, 7(3). http://jasss.soc.surrey.ac.uk/7/3/5.html

Todd, P.M., Billari, F.C., \& Simao, J. (2005). Aggregate age-at-marriage patterns from individual mate-search heuristics. Demography, 42(3), 559-574. http://dx.doi.org/10.1353/dem.2005.0027 
Trochim, W.M.K. (2006). Research methods knowledge base.

http://www.socialresearchmethods.net. Retrieved on 10/07/2014.

Wilensky, U. (1999). NetLogo. http://ccl.northwestern.edu/netlogo. Center for Connected Learning and Computer-Based Modeling. Northwestern University, Evanston, IL.

Wilson, R. (2007). Simulating the Effect of Social Influence on Decision-Making in Small, TaskOriented, Groups. Journal of Artificial Societies and Social Simulation 10(4), 4. http://jasss.soc.surrey.ac.uk/10/4/4.html. Retrieved on 10/07/2014.

Yin, R. K. (2003). Case study research: Design and methods. Applied Social Research Methods Series, Vol. 5, $3^{\text {rd }}$ Ed. Sage.

Journal of Industrial Engineering and Management, 2015 (www.jiem.org)

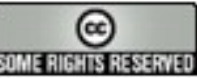

Article's contents are provided on a Attribution-Non Commercial 3.0 Creative commons license. Readers are allowed to copy, distribute and communicate article's contents, provided the author's and Journal of Industrial Engineering and Management's names are included. It must not be used for commercial purposes. To see the complete license contents, please visit http://creativecommons.org/licenses/by-nc/3.0/. 